\title{
Theme: Pediatric Allied Specialities
}

\author{
Gene responsible for increased risk of ear infections \\ in children (Nat Commun. 2016;7:12792)
}

We all know that a very common reason for prescribing antibiotics in children is ear infection - the acute otitis media. Researchers have discovered a gene region that raises the risk of a child having a middle ear infection.

Center for Applied Genomics at The Children's Hospital of Philadelphia (CHOP) in collaboration with University Medical Center, Rotterdam performed a genome-wide association study (GWAS) on two discovery cohorts with DNA samples from 11,000 children. They found that an association between AOM and a site on chromosome 6 containing the gene FNDC1, and then replicated the finding in an independent pediatric cohort with data from 2000 children. The researchers said that although the gene's function in humans has not been well studied, as per studies done in mouse FNDC1 codes for a protein with a role in inflammation.

More specific therapies for the infections in children can be discovered by learning more about the biological interactions between genes and pathogens involved in infections like who are more susceptible to the infections, and where early medical intervention may offer the greatest benefits.

Safety tips for buying toys - prevent blindness USA (http://www.prweb.com/releases/2016/11/prweb 13854122.htm)

Toys are known to cause eye injuries in children if not properly selected. In its recent guideline, Prevent Blindness USA has requested to look for certain safety guidelines while purchasing toys for the children. These are simple, and equally pertinent for our children:

- Don't buy toys that shoot or include parts that fly off, or toys with sharp or rigid points, spikes, rods or edges.

- Choose toys that will withstand impact and not break into dangerous pieces, and be sure toys are suitable for a child's ability and age.

- Don't give toys with small parts to young children, because they tend to put things in their mouths. And avoid toys with long strings or cords, especially for babies and very young children.

- Dispose of uninflated or broken balloons immediately.

- Check the lenses and frames of children's sunglasses; many can break and cause injuries.

- Read all warnings and instructions on the package, and always supervise children and show them how to use their toys safely.

Griseofulvin and Terbinafine are the best antifungals for Tinea capitis (J Am Acad Dermatol. 2016 Nov 2. doi: 10.1016/j.jaad.2016.08.061)

INDIAN PEDIATRICS
Comparative efficacy and safety profiles of systemic antifungal drugs for tinea capitis in children is not clear. To find out the best systemic anti fungal drug, researchers conducted a study using standard Cochrane methodological procedures. Results are interesting, and it was observed that griseofulvin or terbinafine are both effective; terbinafine is more effective for T. Tonsurans, and griseofulvin for $M$. canis infections. Itraconazole and fluconazole are alternative agents, but not optimal choices for Trichophyton infections.

Extra time spent outdoors, when young, can reduce myopia (JAMA Ophthalmol. 2016 Dec 1. doi: 10.1001/ jamaophthalmol.2016.4752.)

World over, myopia is becoming increasingly common, and is associated with potentially sight-threatening complications. Spending time outdoors is protective, but its mechanism is poorly understood. Researchers in Europe tried to examine the association of myopia with ultraviolet B radiation (UVB; directly associated with time outdoors and sunlight exposure), serum vitamin D concentrations, and vitamin D pathway genetic variants, adjusting for years in education.

Increased UVB exposure was associated with reduced myopia, particularly in adolescence and young adulthood. Various theories are being put forward for this finding, but there is no clear explanation. But it is true that kids should spend more time outdoors, with proper safety measures like wearing sun glasses and applying sun screen lotion, to prevent myopia. It is also suggested that being outdoors only affects myopia before it occurs. Once a child needs glasses, it may have no effect on myopia.

Pediatric hypertension is underdiagnosed and undertreated (Pediatrics. 2016;138. pii: e20162195.)

Pediatric hypertension predisposes children to adult hypertension and is an early marker of cardiovascular disease. But how many of us are actually looking for this entity is a big question.

To find out the real numbers of actual treatment commenced for hypertension, a large database of more than 1.2 million children registered under AAP's Collaborative Electronic Reporting Consortium was studied. Only $5.6 \%$ of the children having hypertension were treated with medicines, whereas current guidelines state that children with stage- 2 hypertension or symptomatic stage-1 hypertension should receive antihypertensive medication at the time of diagnosis, and children with asymptomatic stage- 1 hypertension should receive antihypertensive medication if their hypertension persists after 3 to 6 months without pharmacological intervention.

This study motivates us to be more vigilant to diagnose and treat hypertension and pre-hypertension in children.

АмIT P SHAH drnehamit@gmail.com 